\title{
Classification of JERS-1 Image Mosaic of Central Africa using A Supervised Multiscale Classifier of Texture Features
}

\author{
Sassan Saatchi ${ }^{1}$, Franco De Grandi ${ }^{2}$, Marc Simard ${ }^{1}$, Erika Podest ${ }^{1}$ \\ 1. Jet Propulsion Laboratory, 4800 Oak Grove Drive, Pasadena, California \\ 2. MTV, Space Application Institute, Joint Research Center of CEE, Ispra, Italy
}

\begin{abstract}
In this paper, a multiscale approach is introduced to classify the JERS-1 mosaic image over the Central African rainforest. A series of texture maps are generated from the $100 \mathrm{~m}$ mosaic image at various scales. Using a quadtree model and relating classes at each scale by a Markovian relationship, the multiscale images are classified from course to finer scale. The results are verified at various scales and the evolution of classification is monitored by calculating the error at each stage.
\end{abstract}

\section{Introduction}

The large scale mosaic of JERS-1 data over tropical forests during the Global Rain Forest Mapping (GRFM) project has provided the opportunity of applying a series of data analysis and classification schemes for mapping and monitoring land cover and land use (Saatchi et al., 1999). The high resolution imagery and the large scale mosaicking allows the visualization of the landscape at both fine and coarse resolution. In other words, more than one scale of the landscape heterogeneity can be observed or analyzed. Therefore, the data and the image mosaic enhance the traditional photogrametry and remote sensing techniques where one resolution and thus one scale is of ten observed. In this paper, the Central Africa Mosaic is classified for general land cover types such as forest, degraded forest, nonforest, floodplain forest, floodplain low vegetation, grassland savanna, woodland savanna, and water. In order to exploit the landscape multiscale features for extracting thematic information from the JERS-1 mosaic, we have developed a new supervised classifier. The classifier is based on a multiresolution segmentation method that exploits the first order texture features of SAR data at various scales. This method was initially developed for a nonsupervised segmentation approach (Bouman and Shapiro, 1994). In this study, we have modified this concept for a supervised approach by integrating the technique with a maximum likelihood estimation (ML) classifier. The methodology is applied on the Africa mosaic. The classified map is compared with the AVHRR based maps at $1 \mathrm{~km}$ resolution and the Landsat based maps and field data at 100 $\mathrm{m}$. At each scale, the accuracy of maps, differences and complementarities are analyzed.

\section{JERS-1 Africa Mosaic}

In late 1995, the JERS-1 satellite entered into its Global Rain Forest Mapping (GRFM) phase to collect high resolution SAR data over the entire tropical rainforest. SAR data over central and west Africa, from eastern coast of Kenya and Madagascar, to Liberia and Guinea in the west, were acquired from January to March, 1996. The area covered lies between $9^{\circ} \mathrm{N}$ and $9^{\circ} \mathrm{S}$ latitude, extends approximately $6000 \mathrm{~km}$ along the equator, and amounts to about 2200 NASDA processed scenes or 9 million $\mathrm{km}^{2}$. The Congo River basin, about 3.5 million $\mathrm{km}^{2}$, was also covered during the October and November 1996 (the high water season of the river). Madagascar data were acquired in January 1997. The entire data acquired over the African continent have been processed and calibrated. Figure 1 shows the geographical area and the mosaic of JERS-1 SAR data during the GRFM project over Central and West Africa and tropical rain forest region. JERSI SAR is an L-band spaceborne SAR system launched by the National Space Development Agency of Japan (NASDA) in February, 1992. The system operates at $1.275 \mathrm{GHz}$ with horizontal polarization for both transmission and reception. The spatial resolution of the system is $18 \mathrm{~m}$ in 
both arimuth and range. The swath width is $\mathbf{7 5}$ $\mathrm{km}$ and the incidence angle of radar at the center of swath is $38.5^{\circ}$. The single-look images have $4.2 \mathrm{~m}$ pixel spacing in azimuth and $12.5 \mathrm{~m}$ in range and the standard three look image has 12.5 $m$ pixel spacing in both azimuth and range. JERS-I covers the global land surface for several applications such as land survey, agriculture, forestry, fisheries, environmental protection, disaster prevention and coastal monitoring. The satellite flies on sun-synchronous orbits $568 \mathrm{~km}$ above the Earth surface with a recurrent period of 44 days.
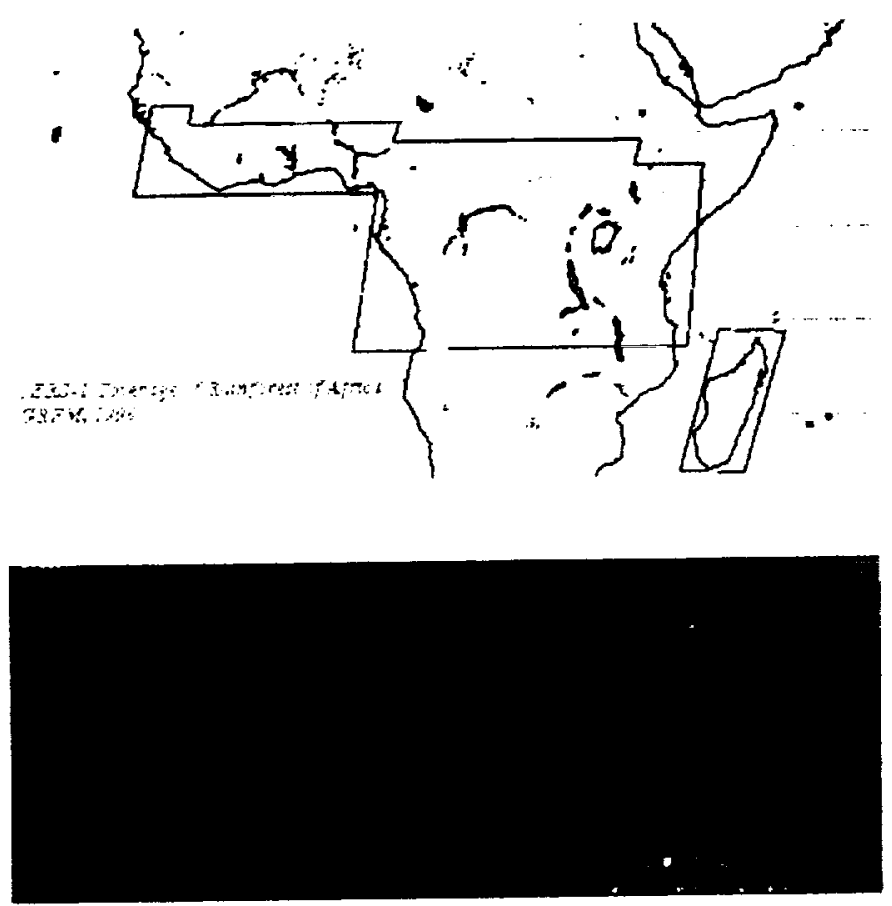

Figure 1. Geographical region of JERS-1 data take during GRFM project and the SAR mosaic image.

\section{Approach}

The objective of using multiscale texture based classification is to segment an image into regions of distinct statistical behavior. An implicit assumption in this procedure is that the statistics of each region are stationary and that each region represents a landscape feature that extends over a significant area (Bouman and Shapiro, 1994). Unlike an unsupervised classification which allows the segmentation based inherent statistics of the image, the supervised approach forces the segmentation to statistics developed by training data sets created over desired land cover types. In a nutshell, the approach includes the following steps:
(1) A sequence of images is produced from JERS1 mosaic where each successive image has half the resolution of its predecessor. These images are texture measures such as mean, coefficient of variation, energy, entropy, variance, contrast, kurtosis, and skewness are developed by using $2 \times 2,4 \times 4,8 \times 8$ windows over 100 meter mosaic image to create $200 \mathrm{~m}, 400 \mathrm{~m}$, and $800 \mathrm{~m}$ images respectively.

(2) A training data set for desired land cover types are created by using a $8 \times 8$ window over the 100 meter resolution mosaic image. The choice of $8 \times 8$ window is dictated by the resolution of the coarsest image and the fact that larger windows may introduced mixed pixel statistics in training data set.

(3) At each scale a supervised maximum likelihood technique is used as a base classifier to classify the image to land cover types. This derived land cover map can be verified by using course vegetation maps available over the continent.

(4) The sequence of random fields or class labels from coarse to fine scale follow a Markov chain. This relation is imbedded in the MLE classifier used at the finer scale which allows segmentation of finer scale images with a priori knowledge of courser resolution classified map.

(5) Class movement and accuracy are monitored at each scale.

To capture mathematically the scale-toscale relationship between classes or image statistics, we use a hierarchical approach known as quadtree model in which a class at scale $n$ is related to its corresponding class at scale $n+1$ by using a Markov chain approach. In other words, assuming that the finest resolution is defined as scale $n=0$, the probability distribution of a class $\omega$ from a set of classes $\Omega$ at this scale can be obtained by the following relationship:

$$
\begin{aligned}
P\left(\Omega^{(n)}\right. & \left.=\omega^{(n)} \mid \Omega^{(l)}=\omega^{(l)}, l>n\right) \\
& =P\left(\Omega^{(n)}=\omega^{(n)} \mid \Omega^{(n+1)}=\omega^{(n+1)}\right) \\
& =P\left(\omega^{(n)} \mid \omega^{(n+1)}\right) \\
P\left(\omega^{(0)}\right) & =\left\{\prod_{n=0}^{L-1} P\left(\omega^{(n)} \mid \omega^{(n+1)}\right)\right\} P\left(\omega^{(L)}\right.
\end{aligned}
$$

where $l$ is scale index, and $P($.$) is the probability$ density function of a class. Given the above 
relationship for class distribution along scales, the MLE distance at cach scale can be modified such that it would include the probability density functions at cach scale:

$$
g_{i}^{(n)}(x)=\ln P\left(\omega_{i}^{(n)}\right)-\frac{1}{2} \ln \left|\Sigma_{i}\right|-\frac{1}{2}\left(x-\mu_{i}\right)^{T} \Sigma_{i}^{-1}\left(x-\mu_{i}\right)
$$

$$
\begin{aligned}
g_{i}^{(n)}(x)= & \ln P\left(\omega_{i}^{(n)} \mid \omega_{i}^{(n+1)}\right)+\ln P\left(\omega_{i}^{(n+1)}\right) \\
& -\frac{1}{2} \ln |\Sigma|-\frac{1}{2}\left(x-\mu_{i}\right)^{T} \Sigma_{i}^{-1}\left(x-\mu_{i}\right)
\end{aligned}
$$

where $g_{i}(x)$ is the distance measured for each pixel $x, I$ and $j$ are the class types and $\mu$ and $\Sigma$ are the mean and covariance vector and matrices developed for each class from the input texture images. Note that at 100 meter resolution or scale $\mathrm{n}=0$, the input image to the MLE classifier is only the backscatter image. In this case, the above relation will take a scalar form.

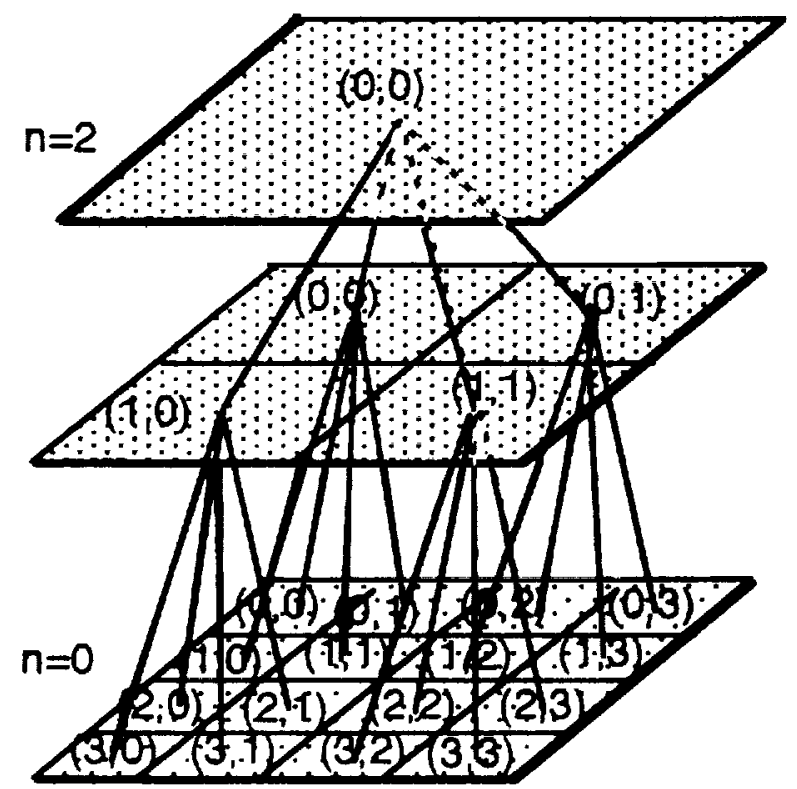

Figure 2. Illustration of multiresolution sequences of three images with pixel-to-pixel quadtree branches.

\section{Discussion}

One of the challenges of employing the MLE approach with the quadtree pyramid approach is to define the transitional probabilities of classes at each scale on the tree structure (the first term in equation 4). Since the transition probabilities are defined on the tree structure, each pixel class is only dependent on a single pixel at the coarser scale. To model the probabilitics in equation (4), we have used an autoregresive type transition function and estimated its cocfficients by using a preliminary fine-to-coarse scale classification (Bouman and Shapiro, 1994; Irving et al., 1997). These log conditional probabilities are computed and stored in image form at each scale. The number of points in the pyramid is approximately $4 N / 3$ where $N$ is the number of pixels at the finest scales. If we choose $M$ as a fixed number of classes for all scales, the total storage required is approximately $4 \mathrm{MN} / 3$.

The procedure shows that the classification at each scale takes into account the information transferred to it by the classification of the previous scale. This method is suitable for large scale JERS-1 mosaic of Central Africa (approximately $35000 \times 25000$ pixel image). At the course resolution, the image size is smaller and therefore the classification and validation is faster. The hierarchical nature of the classifier. insures that at the finer scale the derived land cover map is improved. At each scale the classification can be compared and verified with maps and field information and the resulting map is used as an initial condition at the next finer resolution until individual pixels at 100 meter mosaic are classified. In this study, we compare the results with AVHRR $1 \mathrm{~km}$ land cover map of Central Africa and discuss the accuracy at each stage of classification.

\section{Acknowledgment}

This work is partially performed at the Jet Propulsion .Laboratory, California Institute of Technology under a grand from National Aeronautics and Space Administration.

\section{References}

Bouman, C. and Shapiro, M., 1994, A multiscale random field model for baysian image segmentation, IEEE, Trans. Image Process., vol.3, $162-176$.

Irving, W., Novak, L., Willsky, A., 1997, A multiresolution approach to discrimination in SAR imagery, IEEE Trans. Aeros. Elect. Sys., vol 33, 1157-1169.

Saatchi, S., Nelson, B., Podest, E., and Holt, J., 1999, Mapping land cover types in Amazon basin using $1 \mathrm{~km}$ JERS-1 mosaic, Int. J. Remote Sens., In press. 